\title{
UN RELEVO GENERACIONAL EN \\ LA HISTORIA NATURAL ESPAÑOLA. \\ LA GASCA Y GRAELLS: DEL CIENTÍFICO \\ LIBERAL AL NATURALISTA ISABELINO
}

\author{
Santiago Aragón \\ Université Pierre et Marie Curie (Paris VI), París (Francia)
}

\section{RESUMEN}

El artículo aporta información sobre la formación de Mariano de la Paz Graells en Barcelona, antes de ocupar la cátedra de zoología del Gabinete de Historia Natural de Madrid. Además, se estudia una correspondencia inédita entre Graells y su protector, Mariano La Gasca. Los papeles hablan de nepotismo.

PALABRAS CLAVE: Historia Natural, nepotismo, correspondencia, promoción científica, Mariano de la Paz Graells, Mariano La Gasca.

\begin{abstract}
This article analyzes the Mariano de la Paz Graells' education in Barcelone, before he held the chair of zoology at the Natural History Museum in Madrid. Furthermore, some unpublished letters exchanged between Graells and Mariano La Gasca, his mentor, are studied. The subjet of the correspondence is nepotism.
\end{abstract}

KEY WORDS: Natural History, nepotism, correspondence, scientific promotion, Mariano de la Paz Graells, Mariano La Gasca.

\section{INTRODUCCIÓN}

En 1837 Mariano de la Paz Graells (1809-1898) deja Barcelona y se encamina hacia Madrid, ciudad en la que acaba de ser designado profesor interino de zoología del Museo de Ciencias Naturales. El nombramiento se lo debe 
al botánico Mariano La Gasca (1776-1839)1, entonces profesor de agricultura y director de la Junta del Museo. A este último, su condición de liberal le había costado el exilio en tiempos de Fernando VII. Una vez muerto el monarca, el veterano científico había abandonado su destierro inglés y retomado la vida en España. Durante el viaje de vuelta tuvo ocasión de conocer a Mariano de la Paz, único hijo de su camarada Ignacio Graells (1775-1856), «íntimo amigo desde 1799 $»^{2}$. Tras el fallecimiento de Tomás Villanova (1769-1837), catedrático de zoología del museo madrileño, La Gasca pensó de inmediato en el joven Graells como posible sustituto. Su designación a dedo no fue bien vista por los que durante años habían sido compañeros del finado. Los profesores del Museo consideraban necesaria una convocatoria abierta y la entrada en escena del Recién llegado no estuvo exenta de crítica.

Pese a su larga vida y a su reconocida influencia, Mariano de la Paz Graells continúa siendo un personaje histórico escasamente conocido. Hasta el momento, un muy reducido número de trabajos han abordado de manera exclusiva la figura del naturalista. El primero vio la luz diez años antes de su fallecimiento. Se trata de un artículo inserto en el número 13 de la Revista de Ciencias Naturales cuyo autor, Vila Nadal, era editor de la publicación ${ }^{3}$. Un año después, en 1889, Antonio Elías de Molins incluye una breve biografía y una bibliografía resumida del autor en su diccionario de escritores y artistas catalanes ${ }^{4}$. Tras su muerte, Salvador Calderón, secretario de la Sociedad Española de Historia Natural, le dedicó una nota necrológica de dos páginas en las actas de la institución ${ }^{5}$. Florencio Salamero es el responsable de la primera biografía del naturalista, apenas cinco páginas publicadas en 1907 en la obra Linneo en España ${ }^{6}$, en la que también se incluye otra reseña del personaje

1 Breve perfil biográfico de La Gasca en Puig-Samper, M. A., Maldonado, J. L. y FragA, X. (2004), «Dos cartas inéditas de Lagasca a Humboldt en torno al legado de Mutis», Asclepio, 51 (2), pp. 65-86.

2 YaÑEZ y Girona, A. (1842), Elogio histórico de D. Mariano La Gasca y Segura, Barcelona, Imprenta de Don Antonio Bergnes y compañía, p. 53.

3 Vila Nadal, A. (1888), «Bio-bibliografía del Exc. Dr. D. Mariano de la Paz Graells», Revista de Ciencias Naturales, 13, 2-6.

4 Elías de Molins, A. (1972), Diccionario biográfico y bibliográfico de escritores y artistas catalanes del siglo XIX, Hildestein, Georg Olms, vol. I, p. 669-671.

5 Calderón, S. (1898), «Nota necrológica», Actas de la Sociedad Española de Historia Natural, marzo, 65-67.

6 Salamero, F. (1907), Linneo en España, Zaragoza, Sociedad Aragonesa de Ciencias Naturales, 345-351. 
escrita por Luis Mariano Vidal ${ }^{7}$. Una segunda semblanza fue elaborada por José María Dusmet en sus apuntes para la historia de la entomología españo$1 a^{8}$. Agenjo Cecilia compuso un detallado perfil del científico, rebosante de anécdotas, para el primer número de Graellsia, revista especializada en zoología y editada por el Museo Nacional de Ciencias Naturales desde $1943^{9}$.

La obra botánica de Graells ha sido estudiada por González Bueno y Sánchez Mata ${ }^{10}$.

Más reciente, de 1992, es el texto firmado por José Luis Viejo Montesinos. Dedicado a Graells y a su más popular descubrimiento zoológico, la hermosa mariposa Graellsia isabellae, se incluyó en las páginas de la revista de divulgación científica Quercus ${ }^{11}$, aportación que se completó con una reflexión de Santos Casado de Otaola sobre Graells y las ciencias naturales en España durante el siglo $\mathrm{XIX}^{12}$. Un detallado estudio realizado por Alberto Gomis viene a aumentar el conocimiento del personaje, sobre todo en lo que a los primeros años de su formación respecta ${ }^{13}$. Por su parte, Xosé Fraga se interesa por la trama de interlocutores que Graells tejió en España ${ }^{14}$. Mariano de la Paz Graells cuenta igualmente con una entrada en el Diccionario histórico de

7 Vidal, L. M. (1907), Linneo en España, Zaragoza, Sociedad Aragonesa de Ciencias Naturales, 352-353.

8 Dusmet y Alonso, J. M. (1918), «Apuntes para la Historia de la Entomología de España», Boletín de la Sociedad Entomológica de España, 1, 74-84.

9 Agenjo Cecilia, R. (1943), «Biografía de D. Mariano de la Paz Graells Agüera», Graellsia, 1, 7-21.

10 GonzÁlez Bueno, A. y SÁnchez Mata, D. (1988), «Sobre la obra botánica de M. P. Graells (1809-1898)», Taxon, 37 (1), 151-156.

11 Viejo Montesinos, J. L. (1992), «Biografía de un naturalista y biología del lepidóptero por él descrito. Graells y la Graellsia», Quercus, 74, 22-29.

12 CASAdo de OtaOla, S. (1992), «Graells y las ciencias naturales en la España del Siglo XIX», Quercus, 74, 30.

13 Gomis Blanco, A. (1995), Ciència i tècnica als Països Catalans: una aproximació biogràfica. Als darrers 150 anys, Barcelona, Fundació Catalana per a la Recerca, 119-143. El autor también ha trabajado sobre los catálogos de fauna publicados por Graells. GOMIS BLANCO, A. (2001), «Los catálogos faunísticos realizados en España en la segunda mitad del siglo XIX», Ingenium, 7, 271-284.

14 Fraga, X. A. (1998), «Aportación al estudio de la obra del naturalista Graells», Estudios de Historia de las Técnicas, la Arqueología industrial y las Ciencias, vol. II, Salamanca, Junta de Castilla y León, 839-848; FrAGA, X. A. (2001), «La constitución de la Historia Natural en España. La actividad de los naturalistas a mediados del siglo XIX y el proyecto de catalogación de Graells», Ingenium, 7, 225-252. 
la ciencia moderna en España ${ }^{15}$ y su paso por el Museo de Ciencias Naturales está perfectamente documentado en la obra que Agustín Barreiro dedicó a la institución y de la que han aparecido dos ediciones: una en 1944, prologada por Hernández-Pacheco, y otra en 1992, introducida por Emiliano Aguirre ${ }^{16}$.

Con todo, la mayor parte de la información referente a Graells sigue oculta en los archivos. Ya sea en el Museo Nacional de Ciencias Naturales, en la antigua Universidad Central o en diversas academias, la omnipresencia del naturalista en las instituciones científicas isabelinas fue dejando trazas escritas de su ingente actividad, datos que, poco a poco, salen a la luz ${ }^{17}$.

En el presente trabajo el objetivo fijado es doble. Por una parte, se pretende aportar nueva información acerca de los primeros años de formación de Graells en Barcelona y se relata su paso por la Academia de Ciencias y Artes de la Ciudad Condal. Esta investigación permite apreciar la formación que el naturalista había adquirido antes de dar el salto a Madrid, bagaje intelectual que hizo de él un candidato válido para ocupar la cátedra vacante en la capital. Por otra parte, se estudia una correspondencia inédita mantenida entre Graells y su protector, Mariano La Gasca. Los valiosos documentos se encuentran en el archivo del museo madrileño ${ }^{18}$ y hacen referencia a la promoción de la que el naturalista fue objeto. La rica información vertida en las cartas permite completar las biografías de ambos personajes y tomarle el pulso a la realidad científica de aquel momento. Los papeles hablan básicamente de nepotismo. La transferencia de poder de padres a hijos, o de maestros a discípulos, suele ser considerada causa de estancamiento intelectual, una puerta cerrada a la llegada de aires reformadores. Parece claro que, ante la

15 López Piñero, J. M.; Glick, T. F.; Navarro Brotons, V. y Portela Marco, E. (1983), Diccionario histórico de la ciencia moderna en España, vol. I, Barcelona, Ediciones Península, 423-424.

16 BARreiro, A. J. (1944), El Museo Nacional de Ciencias Naturales, Madrid, CSIC, 81 + 381 páginas; BARreiro, A. J. (1992) El Museo Nacional de Ciencias Naturales (17711935), Aranjuez, Ediciones Doce Calles, 509 páginas.

17 Aragón, S. (2005), El zoológico del Museo de Ciencias Naturales. Mariano de la Paz Graells (1809-1898), la Sociedad de Aclimatación y los animales útiles, Madrid, CSIC, 235 páginas.

18 Salvo indicación contraria, los documentos citados en el presente trabajo se encuentran depositados en el Archivo del Museo Nacional de Ciencias Naturales (AMNCN) y forman parte del fondo Personal Científico, sección Mariano de la Paz Graells, caja 6 (correspondencia con España), expediente 44. También han sido consultados el Arxiu de la Reial Acadèmia de Ciènces $i$ Arts de Barcelona (ARACAB) y el Archivo de la Universidad de Barcelona. El material no se encuentra informatizado ni es consultable a través de internet. 
lógica sucesión de generaciones, el concurso abierto e igualitario es la mejor solución a la hora del relevo, momento en el que los méritos del candidato deben despejar toda posible subjetividad en la designación.

Los datos aportados hacen referencia a dos personajes mayores de la historia natural en el país: Mariano La Gasca y Mariano de la Paz Graells. El tema central de la discusión es la transferencia de poder o, mejor dicho, la promoción del protegido, valor en ciernes, dirigida por su mentor, personalidad ya consagrada. El momento es clave ya que representa la continuidad de dos periodos históricos fundamentales: el último naturalista ilustrado español pasa el testigo al mandarín de la historia natural isabelina:

«Hablaba siempre (se refiere a La Gasca) con especial cariño de nuestros consocios ausentes, amigos íntimos suyos, D. Mariano Graells y D. Eduardo Carreño, y en términos tan expresivos de su talento y constancia, como si éste último debiese ser su sucesor en la botánica nacional, y aquel su representante en la zoología española. Los presentimientos de nuestro sabio moribundo han fallado desgraciadamente en cuanto a Carreño, víctima de su celo y laboriosidad en París, y cuya temprana muerte lloran todos los amantes de las ciencias, iquiera Dios que a lo menos se verifiquen en cuanto a nuestro Graells!» ${ }^{19}$.

\section{LOS AÑOS DE FORMACIÓN EN CATALUÑA}

Mariano de la Paz Graells nació en Tricio (La Rioja) el 24 de enero de 1809. Su padre, Ignacio Graells Ferrer, catalán de Balaguer (Lleida), era médico. Su madre, Antonia Agüera Navarro, era castellana, de Medina de Rioseco (Valladolid) ${ }^{20}$. La familia Graells Agüera pronto se instala en Barcelona, ciudad en la que Mariano de la Paz inicia su formación. Una vez obtenido el título de bachiller en filosofía, en 1827, empieza los estudios de medicina en el Real Colegio de Medicina y Cirugía de la capital catalana ${ }^{21}$. Tras siete años de carrera recibe el diploma de médico-cirujano en 1833. Un año más tarde logra el grado de licenciado en medicina y, en 1835, el de doctor en la misma materia ${ }^{22}$. Estudiante destacado, al final de su formación universitaria se le nombra responsable de la biblioteca del Real Colegio ${ }^{23}$.

19 YAÑEZ Y GIRONA (1842), p. 55.

20 Gomis Blanco (1995), p. 120.

21 Ibidem, p. 120.

22 Archivo de la Universidad de Barcelona. Matrícula de Latinos, libro 4, p. 273.

23 AgenJo (1943), p. 8. 
Durante su etapa catalana Graells cultiva el ejercicio de la medicina, actividad que desarrolla al lado de su cuñado, el doctor Janer ${ }^{24}$. Los biógrafos del naturalista relatan su heroico comportamiento durante la epidemia de cólera que asoló Barcelona en $1835^{25}$, episodio sanitario al que La Gasca hace referencia en su correspondencia ${ }^{26}$. Su creciente reputación jugó en su favor a la hora de ser nombrado director de los baños termales de Puda, en Esparraguera. Desde 1835, y durante doce años, Graells detentó un puesto que finalmente tuvo que abandonar por problemas de compatibilidad con su función docente en la Universidad ${ }^{27}$. Las aguas termales son, como veremos, un tema recurrente en las misivas intercambiadas entre los protagonistas del presente artículo. Con todo, Graells nunca dejó de lado su afición por la historia natural:

«Esta carta instructiva la recibí en Esparraguera, estando rodeado no de mis queridos insectos, distantes entonces cien leguas de mi, sino de una multitud de tísicos, hemoptísicos, herpéticos etc. etc., clientes de mis aguas sulfurosas y que durante la temporada de baños no me dejan distraer tanto como deseara con mis bichos predilectos» ${ }^{28}$.

Esa inclinación por el estudio de la naturaleza Graells la pudo encauzar en los cursos gratuitos impartidos por la Real Junta de Comercio del Principado de Cataluña ${ }^{29}$. Fundada en 1758 por Fernando VI y ratificada en 1760 por Carlos III, la institución había sido creada para defender los intereses de la

24 Biografía de Félix Janer en Chinchilla, A. (1846), Anales Históricos de la Medicina en General. Tomo cuarto. Valencia, imprenta de José Mateu Cervera, pp. 516-522. Edición facsimil (1967), The Sources of Science, 8, Nueva York, Johnson Reprint Corporation.

25 Agenjo (1943), pp. 8-9; Gomis Blanco (1995), p. 124.

26 «Pasado mañana, siendo Dios servido, llegaremos a Montpellier, de donde saldremos el 20 por la noche, y el 22 por la tarde llegaremos a Perpiñán, de donde saldremos el 24 por la mañana si es posible para pernoctar en Figueras, en donde espero adquirir noticias ciertas sobre el estado de la salud de ese pueblo y de los demás de la carrera. Si hay tiempo me alegrará recibir carta tuya en Gerona, noticiándome si podré o no entrar en Barcelona, y en caso de no poder entrar celebraría me dijeses la ruta que debía tomar para libertarme del cólera». Aviñón, 16-12-1834, carta de La Gasca a Ignacio Graells. En la misma cuartilla escribe el 24 de diciembre desde Montpellier. En posdata dice: «Se nos asegura que todo el camino está libre de cólera, ¡ojalá lo esté de facciosos y ladrones! ».

27 Gomis Blanco (1995), p. 124.

28 Carta de Graells a un destinatario desconocido. Madrid, diciembre de 1841. AMNCN, fondo Personal Científico, sección Mariano de la Paz Graells, caja 2 (correspondencia con España), expediente 1.

29 GOMIS BLANCO (1995), p. 120. 
burguesía comercial emergente. Su objetivo era instruir mandos responsables de absorber los progresos tecnológicos que se iban produciendo. La Real Junta era de facto el principal centro de formación profesional de Cataluña. Entre sus muros Graells siguió las formaciones de agricultura y botánica (1825-26 y 1827-28), de física experimental (1826-27) y de química aplicada a las artes (1826-27 y $1827-28)^{30}$. Su vocación naturalista pronto se materializó en comunicaciones escritas, con un primer artículo de tema botánico: un calendario de flora que detalla los periodos de floración de las principales especies vegetales presentes en Barcelona y sus alrededores, trabajo que fue incluido en la edición española de la obra Elements de Botanique de Achille Richard ${ }^{31}$. De manera temprana se manifiesta también su predilección por los insectos y, en noviembre de 1833, se le nombra miembro corresponsal de la Société Entomologique de France, entonces joven asociación fundada el 29 de febrero de $1832^{32}$. Fue presentado en su seno por el barón Feisthamel, socio fundador con el que hacía ya tiempo intercambiaba correos y ejemplares. Además de con su mentor, residente en París, Graells mantenía correspondencia de tema entomológico con otros personajes galos como Companyó ${ }^{33}$, médico, Lévesque en Perpignan y Cailland y Ambiel en Montpellier ${ }^{34}$, personajes sobre los que no se ha podido encontrar información biográfica.

Durante esos primeros años de ejercicio dos instituciones van a desempeñar un papel primordial en la consolidación de las aspiraciones profesionales de Graells. En primer lugar, la Sociedad Barcelonesa de Amigos del País, reestablecida de forma definitiva en 1834, en la que se le nombra secretario de la clase de agricultura. Entre sus colaboraciones figura una memoria dirigida a los viticultores con consignas sobre cómo combatir los gorgojos del género Rhynchites que destruyen los viñedos ${ }^{35}$. El segundo establecimiento clave en el despegue de la carrera científica de Graells fue la Real Academia de Ciencias y Artes de Bar-

30 Ibidem, p. 120.

31 Richard, A. (1831), Elementos de botánica, Barcelona, Imprenta de José Rubio, vol. 2, pp. 209-215.

32 AMNCN, fondo Personal Científico, sección Mariano de la Paz Graells, caja 15 (correspondencia con el extranjero).

33 Lluis Companyó (1781-1871) era cirujano y naturalista. Fue fundador y primer director del museo de historia natural de Perpignan. Es el autor de una historia natural del departamento francés de Pirineos Orientales, obra que se publicó en tres tomos entre 1861 y 1863. Una biografía suya, escrita por Louis Fabre, apareció publicada en 1872 en la revista de la Societé Agricole, Scientifique et Littéraire des Pyrénées-Orientales (vol. XIX, pp. 7-32).

34 AMNCN. Misma signatura.

35 GOMIS BLANCO (1995), p. 123. 
celona. Mariano de la Paz Graells se incorporó a sus filas en $1835^{36}$. Poco antes de salir hacia Madrid, en 1837, Graells presentó públicamente en la corporación sus investigaciones sobre la incidencia de la temperatura en la metamorfosis de los insectos bajo el clima de Barcelona, resultados que fueron más tarde publicados en el boletín de la institución ${ }^{37}$. Una parte del trabajo fue empleada por Graells para elaborar su segunda publicación internacional, un artículo para los anales de la Sociedad Entomológica de Francia en el que detalla la eclosión de los imagos de los coleópteros cebriónidos, adultos que surgen del suelo tras un periodo de lluvias abundantes ${ }^{38}$. Su primer artículo publicado fuera de España fue consecuencia de una comisión encargada por la Real Academia de Medicina y Cirugía de Barcelona. Entre 1830 y 1833 se observó una proliferación anómala de arañas venenosas en los términos municipales de Vendrell y Plá, en el campo de Tarragona, artrópodos que causaban graves problemas de salud pública con sus picaduras. Graells identificó a la viuda negra, y no a la temida tarántula, como la responsable del fenómeno y relacionó la explosión de sus poblaciones con la abundancia de saltamontes en la zona. Sus resultados fueron publicados en el Boletín de la Sociedad entomológica francesa ${ }^{39}$. El fenómeno se reprodujo en 1841, lo que dio pie para que Graells elaborase una cuidada memoria que también tuvo eco internacional ${ }^{40}$.

A estos datos hay que añadirles las precisiones recopiladas por Elías de Molins ${ }^{41}$ sobre la actividad de Graells en la academia barcelonesa antes de su partida, informaciones, en parte inéditas, que se detallan a continuación al mismo tiempo que se citan los documentos manuscritos conservados en el

36 Agenjo (1943), p. 9.

37 Graells, M. P. (1841), «Memoria sobre la influencia de la temperatura baja en la metamorfosis de los insectos y por consiguiente en su aparición», Boletín de la Academia de Ciencias Naturales de Barcelona, 10, 54-60.

38 Graells, M. P. (1837), «Observations sur la cause de l'apparition des Cebrions», Annales de la Société Entomologique de France, 6, 93-99.

39 Graells, M. P. (1834), «Sur les méfaits du Théridion Malmignatte», Annales de la Société Entomologique de France, 3, 26-26.

40 Graells, M. P. (1842), «Notice sur divers faits qui confirment la propiété venimeuse du Latrodectus Malmignatus Walckanaer», Annales de la Société Entomologique de France, 11, 205-219.

41 Memoria del secretario de la Academia de 1898 e historia de la Academia, p. 172. Los listados manuscritos están desarrollados en un texto impreso cuya referencia es: Real Academia de Ciencias y Artes de Barcelona. Nómina del personal académico. Año académico 1908 a 1909. Barcelona [la Academia], 1909, p. 151-177. ARACAB, expedientes de los académicos de la corporación, expediente Elías de Molins 
archivo del centro. El 11 de marzo de 1835 Mariano de la Paz presentó una memoria para ser admitido en la sección de zoología ${ }^{42}$. Su título fue Relación de los insectos que atacan a los olmos, moreras y álamos de los paseos interiores y extramuros de Barcelona ${ }^{43}$. El día 26 marzo fue admitido como académico por unanimidad ${ }^{44}$, leyó su oración gratulatoria el ocho de abril y expuso públicamente su trabajo el 22 del mismo mes. Un tiempo después, el 15 de octubre, se encargó de la cátedra de entomología, desde la que elaboró un trabajo dedicado a los insectos que atacan a los olivos, memoria defendida el 16 de diciembre.

En 1836 su interés parece decantarse por las colecciones. En mayo presenta un roedor procedente de Marañón y pocos días después informa de la existencia de un banco de conchas fósiles, aún sin estudiar, descubierto por él mismo en Montjuich. En junio regala una tenia conservada en alcohol, varios bivalvos de río americanos y cinco minerales del país. Su evidente interés por las producciones de la naturaleza hace que, el 22 de julio de 1836, se le designe conservador de la colección de la Academia y más tarde, el 26 de octubre de 1836, se le encargue crear un gabinete de historia natural en el seno de la institución. En 1837 dona un zoófito y un mustélido (19-01-1837), lee la referida memoria sobre la influencia de las bajas temperaturas en la metamorfosis de los insectos (03-05-1837) y discute sobre los estragos que la tisis tuberculosa causa entre los monos que se crían en Europa (16-05-1837), ponencia que apuntala con la presentación de un pulmón encharcado de primate. En julio de 1837 ocupa el puesto de bibliotecario y en septiembre se responsabiliza de la cátedra de zoología, progresión de cargos que únicamente se verá interrumpida por el anuncio de su interinidad en el museo madrileño, noticia que él mismo hace pública el 20 de diciembre de $1837^{45}$.

Desde 1835 Mariano de la Paz Graells se encargaba de los cursos de zoología y taxidermia en la academia, con 17 alumnos inscritos en 1835-36 y

42 Cartas de Mariano de la Paz Graells. Barcelona. (1) 20-02-1835. Dirigida a Antonio Monmany. Carta que acompaña una memoria sobre los insectos que atacan a los olmos. El envío incluye una caja entomológica con los referidos insectos. (2) 25-02-1835. Carta dirigida a la Academia con el mismo fin y en las mismas condiciones. (3) Sin fecha. Graells solicita su ingreso en la Academia. ARACAB, expedientes de los académicos de la corporación, expediente Mariano de la Paz Graells.

43 Carta de Yáñez. Barcelona, 07-03-1835. Informe muy favorable al ingreso de Mariano de la Paz Graells en la Academia. ARACAB, mismo expediente.

44 Carta de Juan Ma Foix. Barcelona 11-03-1835. El firmante, antiguo profesor de Mariano de la Paz en el Colegio de Cirugía, le recomienda para su ingreso. ARACAB, mismo expediente.

45 Carta de Mariano de la Paz Graells. Barcelona 20-12-1837. Corre unida una carta de felicitación de la Academia, sin autor, con fecha de 21-12-1837. ARACAB, mismo expediente. 
sólo cinco en 1836-37. Durante el curso 1837-38 la asignatura no se impartió, posiblemente a causa de la partida de Graells. En el curso 1838-39, Llobet, profesor de mineralogía y geología, se hizo también cargo de la zoología, mientras que la taxidermia fue impartida por Bosés. Ambos instruyeron a un único alumno inscrito, razón por la que a partir de 1839-40 la materia desaparece del plan docente de la institución ${ }^{46}$.

Tras su nombramiento en Madrid Graells no interrumpe el vínculo con la corporación catalana. De hecho, en su carta de 20 de diciembre de 1837, deja claro que asume sus responsabilidades en la capital del Estado pero sin renunciar a su cátedra barcelonesa. Estas fueron las noticias sobre Graells recibidas a partir de entonces en la academia de Barcelona ${ }^{47}$ : el seis de junio de 1838 avisa de que ha revisado el herbario de Pavón adquirido por la academia y dice que piensa adquirir el de Barnades para cederlo a la institución, además de regalar 500 especies de plantas recogidas en los alrededores de Madrid; el 15 de junio de ese mismo año la academia, a propuesta de Graells, acuerda la adquisición del manuscrito y las láminas de la obra Laurographia de Pavón; el ocho de agosto hace personalmente entrega de 40 cortezas de quina traídas de Madrid, de las lecciones originales manuscritas de la botánica de Quer y de un libro con los nombres castellanos y americanos de las plantas de Perú, Chile y Quito regalado por Pavón; el 27 de marzo de 1839 Yáñez comunica el éxito obtenido por Graells en Madrid con las primeras vivisecciones practicadas en España en cátedra pública; el 27 de marzo, y gracias a la mediación de Graells, se reciben en Barcelona piezas duplicadas del museo de ciencias naturales de la capital; el 21 de mayo Yáñez da parte de las demostraciones hechas por Graells sobre la anatomía comparada del caracol y el 12 de junio el socio Rosés da cuenta de otras relativas a la «inhibición o empapamiento y trasudación de los tejidos animales»; el 23 de octubre Graells anuncia por escrito el descubrimiento de nuevas especies de insectos en los alrededores de Madrid y el 27 de noviembre dice haber sido el primero en usar el daguerrotipo en la capital; el 12 de febrero de 1840 Yáñez detalla el descubrimiento hecho por Graells de una nueva especie de murciélago y el 16 de febrero de 1842 dice que el botánico Boissier ${ }^{48}$ ha dedicado a Mariano de la Paz un nue-

46 Matrículas de estudios. Cursos 1835-36 a 1846-47. ARACAB, caja 341.

47 Listado de la actividad de Graells en la Academia (1838-98). ARACAB, expedientes de los académicos de la corporación, expediente Mariano de la Paz Graells.

48 Edmond Boissier (1810-1885), botánico suizo, parece ser que no llegó a conocer personalmente a Mariano de la Paz Graells, al menos no le cita en el prólogo de su obra dedicada a la flora del sur de España (BOISSIER, E. (1839), Voyage botanique dans le midi de l'Espagne pen- 
vo género de planta crucífera. En distintas fechas (25-04-1847, 01-03-1855, 30-06-1875, 29-01-1898) Graells remite ejemplares de sus publicaciones a la academia. El 28 de febrero de 1898 se da parte de su fallecimiento.

\section{CORRESPONDENCIA ENTRE LA GASCA Y LA FAMILIA GRAELlS}

La carta más antigua intercambiada entre Mariano La Gasca e Ignacio Graells está fechada en Madrid un 19 de enero de 1801, unos años antes del nacimiento de Mariano de la Paz. En la misiva la figura de éste parece estar ya presente de una manera implícita:

«Celebro que nuestro Pozo sea ya padre de una tan hermosa niña y me alegraré lo seas tu ya de un gracioso infante que sea la esperanza y apoyo de padres tan dignos. (...) Anima a tu amada esposa y dila (sic) que si pare un niño lo hemos de casar con la niña que haré presto a mi futura esposa» ${ }^{49}$.

El origen de la estrecha relación entre Ignacio Graells y Mariano La Gasca no está claro aunque, como ya ha sido dicho, Agustín Yáñez y Girona habla de una amistad íntima surgida en $1799^{50}$, vínculo que se prolongará en la figura de Mariano de la Paz que cuenta con la protección y el consejo de La Gasca, su «segundo padre» $»^{51}$.

La mayor parte de las cartas conservadas en el archivo del museo madrileño son posteriores a la primavera de 1834 , momento en el que el botánico inicia los preparativos para su regreso a España.

«En Inglaterra he escrito bastante, he recogido algún herbario (...); tendré como unos cuatrocientos volúmenes, la mayor parte remitidos de España, resto de mi antigua librería, parte de mi antiguo herbario, y una porción de espigas y aun paja, que me podrá ser útil. (...) a lo menos doce o catorce cajones grandes, y dos o tres baúles de ropa y manuscritos. Sabes que en Sevilla perdí lo más precioso que tenía (...), y sería una gran desgracia perder ahora el resto y sobre todo los dibujos y manuscritos sobre los cereales, obra que a pesar de mis circunstancias y de mi quebrantada salud, no dejaré de las manos mientras viva. (...) estoy casi decidido a pedir un retiro y suponiendo sea al menos de unos 15000 reales, deseo saber si podría

dant l'année 1837, Paris, Gide et cie, vol. I, 248 pp.). si que llegó a conocer a Miguel Colmeiro y a Mariano La Gasca, quien probablemente le hablaría favorablemente de su discípulo.

49 Carta de La Gasca a Ignacio Graells. Madrid, 19-01-1801.

50 YAÑEZ Y GIRONA (1842), p. 53.

51 Carta de La Gasca a Mariano de la Paz Graells. Madrid, 30-05-1835. 
subsistir en esa ciudad (...). Debes tener entendido que abandoné la medicina y no me atrevo a recetar agua de malvas a mi criada $\rangle^{52}$.

«Yo entro en España a cierraojos, pero no sin grandes temores de no pasarlo $\tan$ bien como en Inglaterra» ${ }^{53}$.

La Gasca se erige en mentor del joven Graells y manifiesta la clara intención de afianzar el gusto que su protegido poseía por la historia natural. La pasión naturalista de Graells encontró un primer terreno de experimentación en la botánica y, como ya ha sido dicho, con 22 años publicó su calendario de flora barcelonesa ${ }^{54}$. La Gasca no descuidó su formación en este aspecto y le instruyó acerca del método natural de clasificación de los vegetales:

«El estudio de las relaciones naturales de las plantas es muy difícil, y su verdadero aprecio está reservado a pocos, muy pocos (...). Hoy día es cierto aún que no está descubierto el método natural; pero sin embargo se conocen bastante bien retazos, fragmentos u órdenes, cuyo conocimiento ha producido ya grandes utilidades (...). Como no hay principios ciertos que nos conduzcan al conocimiento de las plantas según el método natural, nos es preciso valernos en los principios de los métodos artificiales, y después de encontrado el nombre de las plantas ver el lugar que ocupan en el de Jussieu, y mejor aún en la obra de De Candolle (...). Sin embargo esto no es bastante, y así no satisface enteramente el espíritu, porque en los libros se presentan los seres en una serie lineal, que ciertamente no es el orden que sigue la naturaleza, sino en forma como de mares tanquam territorium in mappa geographica, según dijo sabiamente Linneo. (...) Todos hablan con entusiasmo del método natural, como si estuviese descubierto ya, y no lo extraño, porque yo que estoy persuadido de lo contrario, al hablar de él me ilusiono hasta el punto de hablar de él como si lo viera establecido. Por eso me figuro yo que el método natural es una bella extremada que nadie ha visto en la realidad; unos la vieron un ojo, poquísimos vieron los dos, otros describieron una mano, otros han visto los dedos del pie etc., y como las partes vistas aparecieron sumamente perfectas, de ahí es que infirieron la perfección del todo que nadie vio aún. ¿Querrás creer que después de tantos años de estudiar los cereales tengo grandes dudas que tal vez no podré vencer sobre los verdaderos límites de las especies? Acaso moriré antes de poder resolver a mi satisfacción ese problema. (...) Por eso te encargo mucho, me recojas cuantos cereales puedas en la cosecha próxima en los campos de esos alrededores,

52 Carta de La Gasca a Mariano de la Paz Graells. Saint-Heliers (Jersey), 18-04-1834.

53 Carta de La Gasca a Ignacio Graells. Aviñón, 16-12-1834.

54 Graells, M. P. (1831), «Calendario de flora o épocas de la florescencia de algunas planats bajo el clima de Barcelona». En: A. Richard, Elementos de botánica, Barcelona, imprenta de José Rubio, tomo 2, pp. 209-215. 
y que hagas iguales encargos a tus amigos y a tu padre (...), pues yo no abandono esta obra mientras viva» ${ }^{55}$.

De manera recurrente La Gasca no deja de expresar su desconfianza en la ciencia española, fundamentalmente en lo que respecta a la reputación de los naturalistas, colectivo desprovisto de todo tipo de amparo oficial. Preocupado por el futuro de Graells, el botánico le insiste para que nunca abandone la práctica médica, único medio de asegurarse un bienestar material en un país acostumbrado a menospreciar la actividad intelectual:

«¡Mírate en mi espejo! Al cabo de mis días y de ... sólo me ofrecen 15000 reales que un médico suele ganar acaso en un día. Los mismos médicos dirán que eres un buen herbolario, y por lo mismo mal médico. Toma la botánica como un adorno y un auxilio para ejercer dignamente la facultad de curar, que es la que da dinero. La Botánica en un siglo no dará sino pesares al que tenga la desgracia de sobresalir en ella en España» ${ }^{56}$.

La desconfianza de La Gasca en ese aspecto permanece incluso después de confirmarse el nombramiento de Graells como profesor de zoología y taxidermia en la Academia de Barcelona lo que, en principio, paracería otorgarle reconocimiento social y cierta estabilidad profesional:

«Celebro verte ya de profesor público de Zoología, pero quisiera verte al menos con una dotación de mil duros; pero temo mucho que tardará el tiempo para mi tan deseado de ver honrados y bien remunerados los profesores públicos de ciencias útiles. (...) No abandones de modo alguno el ejercicio de la medicina y cirugía» ${ }^{57}$.

«Mucho me alegrará verte director de los baños de Esparraguera, porque creo han de ser más productivos que una cátedra de ciencias naturales, (...) celebro verte enseñando Zoología, y lo celebraría más si me añadieses que por semejante cargo te daban 20.000 reales anuales; enseñas de balde y además de poner el paño tendrás que añadir el hilo y cuanto se necesite. (...) Yo en tu edad estaría tan ufano como tu estarás, y te aseguro que el día en que di la primera lección pública en 1804, no me hubiese cambiado por el Emperador de la China; pasión noble que ocupa el corazón de un joven, pero desgraciadamente mal apreciada por la generalidad que nunca sabe agradecer lo mucho que ella vale: esta triste verdad es la que se saca después de muchos desengaños. (...) Si regalan alguna ave etc. para ese proyectado Museo (se refiere al de la Academia de Barcelona), pondrás al pie de él

55 Carta de La Gasca a Mariano de la Paz Graells. Saint-Heliers (Jersey), 18-04-1834.

56 Misma referencia.

57 Carta de La Gasca a Mariano de la Paz Graells. Madrid, 03-12-1835. 
regalado por D. F. de tal, preparado y denominado gratis (palabra subrayada tres veces) por D. M. Graells, al menos que te quede ese honor ${ }^{58}$.

La Gasca conocía bien la realidad científica del país y había sufrido las consecuencias de los vaivenes políticos del inestable periodo que le tocó vivir. Nombrado director del Jardín Botánico de Madrid tras la Guerra de la Independencia, su talante liberal y su puesto de diputado en Cortes durante el Trienio Liberal le abocaron el exilio una vez restaurado el absolutismo. Desde 1823 el botánico residió en Londres y Jersey y sólo pudo volver al país tras la muerte de Fernando VII. Esa experiencia personal justifica su desconfianza en instituciones y gobiernos:

«Yo no concurro a la Sociedad (probablemente la Sociedad Barcelonesa de Amigos del País) porque no tengo tiempo ni devoción. Y no tengo devoción porque en semejantes corporaciones españolas se habla mucho y se hace poco, y se agradece menos al que algo hace ${ }^{59}$.

«Mucho celebro los progresos de esa Academia (se refiere a la Real Academia de Ciencias y Artes de Barcelona) que los hará ínterin el Gobierno no meta su mano destructora y abrasadora» ${ }^{60}$.

De hecho, la actualidad social del país es tema frecuente en sus misivas, como corresponde a la inquietud de un personaje liberal con participación activa en la política de su tiempo, interés que poco a poco deriva hacia el desengaño y el olvido voluntario:

«(...) tenía y tengo grandes temores de que se generalice la guerra civil, que, si por desgracia, llega a verificarse, dejará asolada nuestra desgraciada patria. (...) Quiera Dios dar juicio a los buenos españoles, y que no intenten propasarse más allá de los justos límites, que sería el modo de perderlo todo. (...) se me devolvió la cátedra, pero con 15000 reales anuales, o sea con 9500 reales menos de lo que tenía en 1820 (...). Ya ves el afrancesado Burgos ${ }^{61}$, que tal se porta con el patriota La Gasca; pero él hace bien de vengarse ya que lo tiene en su mano, y los cabrones

58 Carta de La Gasca a Mariano de la Paz Graells. Madrid, 03-01-1836.

59 Carta de La Gasca a Mariano de la Paz Graells. Madrid, 30-05-1835.

60 Carta de La Gasca a Mariano de la Paz Graells. Madrid, 01-08-1836.

61 Se trata de Javier de Burgos Olmo (1778-1849), secretario de Estado de Fomento durante las presidencias de Cea Bermúdez y Martínez de la Rosa, cargo desde el que estableció la división territorial de España por provincias. URQUIJO Y GoITIA, J. R. (2001), Gobiernos y Ministros Españoles (1808-2000), Madrid, CSIC, p 37. 
españoles sufren el dominio de un traidor y algo más. Pero estamos en los principios de una nueva carrera y hay mucho que ver» ${ }^{62}$.

«No soy yo sólo el que cree y dice que no hay Nación; lo dicen otros que no han estado emigrados pero que se han visto abandonados por ella, y por fin lo dicen los hechos» ${ }^{63}$.

«Hace sobre 3 meses que no he leído un periódico, pero he recogido una cosecha de unas dos mil castas de cereales. Así no se engañaba el que escribía que sólo vivía entre los trigos. Viviendo así, y ajeno de la política, he recobrado mi salud y estoy haciendo un bien a la ciencia y a mi Patria» ${ }^{64}$.

\section{LA PROMOCIÓN DEL PROTEGIDO}

Pese al desánimo manifiesto en lo tocante a los asuntos políticos y científicos de España, La Gasca no descuidó su compromiso con Mariano de la Paz Graells y, como su «segundo padre», una vez recuperada parte de su influencia se encargó de situar al joven científico en el seno del principal organismo dedicado al cultivo de la historia natural en el país. Eso si, no sin antes advertirle de que, en Madrid, la ciencia no estaba mejor pagada:

«Es necesario que sepa Mariano que deben trece meses a los del Gabinete de Historia Natural y doce al Jardín Botánico, y que para el año siguiente hay muy mala perspectiva. Tiene mi casa y la oferta de que el día que no haya de comer (como ya ha sucedido el mes pasado), el hambre repartido entre más tocará a me$\operatorname{nos} »^{65}$.

Tras su regreso a Madrid, en 1835, La Gasca se había ocupado de la enseñanza de la agricultura en el Gabinete de Historia Natural ${ }^{66}$ y desde ese mismo instante había iniciado las acciones encaminadas a la promoción de Mariano de la Paz:

62 Carta de La Gasca a Ignacio Graells. Saint-Heliers (Jersey), 18-04-1834.

63 Carta de La Gasca a Mariano de la Paz Graells. Madrid, 30-05-1835. En su comentario La Gasca se está refiriendo a la división de España en dos bandos: carlistas y cristinos, enfrentados en guerra tras la muerte de Fernando VII y la designación de su hija Isabel como legítima sucesora al trono. En la primavera de 1835 se produjo un corto repunte de la causa carlista sobre todo en Cataluña, Castilla la Nueva y Extremadura. MARTíneZ DE VelasCO, A. y SÁNCHEz Mantero, R. (1997), Historia de España, Madrid, Espasa, vol. X, p. 153.

64 Carta de La Gasca a Mariano de la Paz Graells. Madrid, 01-08-1836.

65 Carta de La Gasca a Félix Janer. Madrid, 28-09-1837.

66 BARReiro (1992), p. 169. El resto de profesores eran: Andrés Alcón de química, Donato García de mineralogía y Tomás Villanova de zoología. 
«Anteanoche hablé con D. Ángel Vallejo ${ }^{67}$, y me prometió que dentro de ocho o diez días determinaría sobre el museo (...). Le hablé en tu favor, y otro amigo le había hablado antes en el mismo día. Pero no por eso confíes en nada; porque juzgo que en la actualidad los hombres que no son intrigantes no pueden esperar mucho. Sin embargo se me ha ocurrido que podría ser útil me remitieses una representación documentada con la relación de tu carrera literaria, pidiendo una plaza de Profesor de Zoología en el Museo de Ciencias Naturales de Madrid, y otra también documentada pidiendo la plaza de viceprofesor del mismo ramo. Tú debes suponer que si puede ser alcanzar la primera, no pediré la segunda» ${ }^{68}$.

La estrategia concebida por La Gasca pasa por activar las publicaciones científicas de su pupilo, al que constantemente insta para que envíe a la imprenta los resultados de sus investigaciones ${ }^{69}$. También quiere facilitarle la tarea en sus pesquisas y se ocupa de dotarle del material necesario para su adecuado progreso. Así, en una de las cartas anuncia el envío de un microscopio, de un spelling book y de un cuaderno titulado Nixus plantarum, obra del botánico inglés John Lindley (1799-1865) ${ }^{70}$. La decisión del gobierno de suprimir las plazas de director en los baños termales del país, y de transferir al mismo tiempo la gestión de los balnearios a las diputaciones provinciales ${ }^{71}$, será un detonante que intensificará el intercambio epistolar entre ambos y pondrá a prueba las capacidades de Graells. La Gasca quiere que Mariano de la Paz se convierta en ferviente opositor a tal medida y, para ello, le pide recoja toda la información que esté a su alcance sobre los baños de aguas minerales. Hace especial hincapié en la rica documentación inédita atesorada por su padre, antiguo director del establecimiento de Caldas de Montbuy ${ }^{72}$. La

67 Debe tratarse de Angel Vallejo Villalón, ministro de Interior interino entre 28-08-1835 y 15-09-1835, durante la presidencia de José María Queipo de LLano, conde de Toreno. URQUIJO Y GOITIA (2001), pp. 37 y 333.

68 Carta de La Gasca a Mariano de la Paz Graells. Madrid, 26-05-1835.

69 «Mi querido Mariano: recibí la tuya con el extracto y lámina de Rhynchites betuleti y por mi anterior conocerás cuanto me habré alegrado de ver tu nombre volar en letras de molde» (30-05-1835); «Me alegro de la Cabera graellsiana porque da honor, y acaso podrá abrir el camino para llegar a obtener un pedazo de pan aunque sea escaso» (03-01-1836); «(...) necesario es que te des a conocer con algún trabajo útil; tal sería la descripción de los insectos que atacan los cereales» (01-08-1836); «Mucho celebro tus tareas entomológicas, pero quisiera yo ver lo que has publicado en Francia, para publicarlo aquí como copiado o traducido del papel francés» (12-01-1837). Cartas de La Gasca a Mariano de la Paz Graells, Madrid.

70 Carta de La Gasca a Mariano de la Paz Graells. Madrid, 26-06-1835.

71 Carta de La Gasca a Mariano de la Paz Graells. Madrid, 30-11-1836.

72 Carta de Francisco La Gasca (en nombre de su padre convaleciente) a Mariano de la 
poca diligencia con la que el joven Graells se ocupó del asunto no fue del agrado de su protector y dio lugar a airadas contestaciones por parte de este:

«Mi señor don Mariano el de los sesos magullados ¿cree usted en conciencia que ha cumplido usted con mi encargo de remitirme noticias todos los correos sobre aguas minerales con sólo las escasísimas noticias que remitió usted en una sola carta al amigo Roses y las que en la carta de ayer me remite? (...) tu puedes alargar cuanto quieras, pues si en este momento te tuviera aquí te daría una paliza, y por las sesiones que habrás visto en el Diario de las Cortes podrás conocer que la merecías muy bien (...). Tu padre sin duda tiene un tesoro que puesto en circulación ahora hubiese venido muy bien, pero como lo tiene escondido para nadie vale» ${ }^{73}$.

«Mi querido almita de mierda: ¿con que ha sido necesario que un pobre moribundo te haya dado una paliza para poder despertar? ¿No hubiera sido mejor que hubieses despertado dos meses ha al primer aviso? Despiertas cuando ya casi está pasado el nublado» ${ }^{74}$.

El 21 de septiembre de 1837 el museo cambió de régimen. Por Real decreto quedó suprimida la Junta de Protección y se creaba en su lugar una Junta Gubernativa formada exclusivamente por profesores del establecimiento. La asamblea estaba dirigida por uno de ellos elegido por votación ${ }^{75}$ y La Gasca fue nombrado presidente de la misma, lo que le colocaba en una situación de poder privilegiada ${ }^{76}$ :

« (...) la provisión de la cátedra ya no depende sólo del ministro y de mí, sino felizmente para la ciencia, de la Junta de Profesores que se instalará pasado mañana: sin embargo tentaremos la interinidad. Si el tal Mariano me hubiese tenido armado con documentos, según se lo insinué varias veces, probablemente hubiera sido catedrático antes de la existencia de la Junta de Profesores, así que culpe a su pereza o descuido. (...) todo lo que usted habrá leído es obra mía y del ministro: aproveché la amistad y los momentos. Sin embargo esta noticia es sólo para usted y la familia. Usted conocerá cuan sensible me será no haber podido hacer más por Mariano, de lo que él tiene la culpa, según dije antes, si lo cogiera ahora, en este momento, le diera un no sé qué: me ha privado del placer mayor, después del que he tenido en emancipar del yugo de la ignorancia las ciencias naturales y sus profesores $\rangle^{77}$.

Paz. Madrid, 20-12-1836. Elías de Molins, en 1889, hace referencia a ese material y especifica, en nota a pie de página, que los escritos inéditos se encuentran en poder de Mariano de la Paz Graells. EliAS DE Molins (1972), vol. I, p. 668.

73 Carta de La Gasca a Mariano de la Paz Graells. Madrid, 12-01-1837.

74 Carta de La Gasca a Mariano de la Paz Graells. Madrid, 10-02-1837.

75 BARREIRO (1992), p. 175.

76 YAÑEZ Y GIRONA (1842), p. 51.

77 Carta de La Gasca a Félix Janer. Madrid, 28-09-1837. 
Escasamente un mes antes de constituirse la citada Junta Gubernativa había fallecido Tomás Villanova, profesor de zoología del museo. La cuestión de su sucesión aparece ya evocada en la primera reunión del órgano de gestión, una sesión presidida por La Gasca. En ese instante la figura de Mariano de la Paz Graells aparece en la escena madrileña por primera vez ${ }^{78}$. Junto a él compite por la vacante Anastasio Chinchilla (1801-1867) ${ }^{79}$, cirujano del hospital militar que ya había sustituido a Villanova en el museo durante la enfermedad de este último ${ }^{80}$. Se presenta como su legítimo sucesor e incluso se ofrece para enseñar gratuitamente con una interinidad ${ }^{81}$. La decisión final de la Junta es la de ofertar dos plazas de catedrático, una dedicada a la anatomía comparada de los animales vertebrados y otra a la de los invertebrados, puestos que en principio se ofrecen en interinidad para posteriormente conceder la propiedad según el desempeño ${ }^{82}$.

La influencia de La Gasca y sus contactos le permiten seguir adelante en la promoción no sólo de su protegido, sino también de su propio hijo ${ }^{83}$. Con todo, de las palabras del botánico se deduce que Graells partía en desventaja, en gran medida debido a su dejadez a la hora de dar respuesta a los requerimientos de su mentor y a ciertos errores de bulto en su producción científica:

«Encontré dos ejemplares del extracto de tu memoria sobre el Rinchetes betuleti y haré se presente uno en la Junta para reunirlo a tu representación. Me olvidé decirte el gran disparate cometido en la lámina que representa el pámpano de la vid

78 Carta de La Gasca a Mariano de la Paz Graells. Madrid, 03-10-1837.

79 Anastasio Chinchilla Piqueras es autor de Anales históricos de la medicina en general y biográfico-bibliográficos de la española en particular (1841-1846). Natural de Ayora, estudió medicina en Valencia. Durante el curso académico 1836-37 siguió una formación en zoología en el museo de Madrid a cargo de Tomás Villanova. SAlCEDO y GinESTAL, E. (1904), El doctor Chinchilla. Estudio biográfico, bibliográfico y crítico, Madrid, imprenta de Ángel B. Velasco, p. 8; GuERRA, F. (1967), Introduction to the works of Chinchilla and Morejón. The Sources of Science, 8, p. XIV.

80 BARREIRO (1992), p. 175.

81 Carta de La Gasca a Mariano de la Paz Graells. Madrid, 06-10-1837.

82 Misma referencia.

83 «Ya ves que dejó de ser ministro mi amigo (se trata de Diego González Alonso, ministro de la Gobernación entre 23-08-1837 y 01-10-1837, durante la presidencia de Joaquín Baldomero Fernández Espartero, conde de Luchana; URQUIJO GoITIA (2001), pp. 39 y 229) pero felizmente hizo antes lo que yo tanto deseaba, y además nombró a tu tocayo Mariano oficial $4^{\circ}$ de la Biblioteca pública de San Isidro con cinco mil reales anuales. (...) Supongo que a nadie dirás cosa alguna del contenido de ésta sino a los de la familia. Ahora conocerás las gravísimas faltas que has cometido contra ti mismo en no hacer varias de las cosas que te he advertido en las mías». Misma referencia. 
con los zarcillos que salen del pecíolo de la hoja, siendo así que siempre nacen del punto opuesto a ella. Además la disposición de los nervios de la hoja es pésima, y el tallo se representa casi recto, cuando es flexuoso. Yo creo que el pintor hizo el dibujo de memoria, y tú no echaste de ver tantas y tamañas imperfecciones, que a los ojos de los inteligentes te representarán como absolutamente ignorante en Botánica. En todo se necesita reflexionar» ${ }^{84}$.

El resultado fue finalmente el deseado y Mariano de la Paz Graells fue nombrado catedrático interino de zoología en noviembre de 183785 . Sus inicios en el museo madrileño no iban a ser fáciles, como bien vaticinaba $\mathrm{La}$ Gasca: «Yo no se cómo, se ha entendido tanto que yo lo protejo (...), que acaso esa misma opinión le perjudique» ${ }^{86}$.

En el museo madrileño se conserva el expediente que Graells presentó con la intención de que se le conmutase la cátedra de zoología en la academia de Barcelona por la misma en la capital ${ }^{87}$. Los documentos los remite, el 16 de noviembre de 1837, José Moreno de la Güera (sic), primo de Graells, y se los envía al secretario de Estado del despacho de gobernación de la Península. El conjunto de papeles se organiza en cuatro carpetas, identificadas de la A a la $\mathrm{D}$, que incluyen cartas originales, copias legalizadas de cartas u oficios, certificaciones legalizadas y relación de méritos.

Las cartas autógrafas de la carpeta A (entre paréntesis se indica la fecha y el lugar de expedición de cada misiva) están firmadas por: Barker Webb ${ }^{88}$ (Valencia, 03-06-1826), Companis ${ }^{89}$ (Perpiñán, 07-03-1834), Angel Boissy ${ }^{90}$ (París, 20-04-1835), Duponchel ${ }^{91}$ (París, 20-11-1835), Leon Dufour ${ }^{92}$ (Saint

84 Carta de La Gasca a Mariano de la Paz Graells. Madrid, 24-10-1837.

85 Carta de La Gasca a Ignacio Graells. Madrid, 05-12-1837.

86 Misma referencia.

87 AMNCN, fondo Personal Científico, sección Mariano de la Paz Graells, caja 43 (actividad docente).

88 Philip Barker Weeb (1793-1854), botánico británico, recorrió gran parte de Europa en sus expediciones. En Cataluña estuvo en 1826, fecha en la que conoció a Graells cuando éste sólo contaba diecisiete años. El joven Mariano de la Paz le acompañó en sus excursiones por Caldes de Montbui, las riberas del Besòs, Cardona y Montserrat. Su primera contribución a la flora ibérica se publicó en París en 1838 con el título de Iter Hispaniense [CAMARASA, J. M. (1989), Botànica i Botànics dels Països Catalans, Barcelona, Enciclopèdia Catalana, p. 117-118]

89 Se presenta como zoólogo distinguido.

90 Idem.

91 Philogène Auguste Joseph Duponchel (1774-1846) era militar aficionado a la entomología y especialista en mariposas. Continuador de la Histoire Naturelle des Lépidoptères de France, obra iniciada por Jean-Baptiste Godart, él es el autor de 12 de los 17 volúmenes aparecidos entre 1832 y 1842 con descripciones de más de cuatro mil especies. Participó en la 
Sever, 16-12-1837), Lefebvre93 (París, 27-12-1835) y Pedro Giralt ${ }^{94}$ (Montevideo, 03-05-1837). La carpeta B contenía copias legalizadas de cartas del secretario de la Sociedad Entomológica de Francia, Alexandre Lefebvre, quien comunica que Graells ha sido nombrado miembro de la asociación (París, 08-111833), de Hope, presidente de la Sociedad Entomológica de Londres (Londres, 07-08-1835), de Voitel (Soleure, Suiza, julio de 1837) y de oficios de la Real Academia de Medicina y Cirugía de Barcelona (Barcelona, 19-07-1833), de la Sociedad económica de amigos del País de Tortosa (Tortosa, 14-10-1835) y de la Academia de Ciencias y Bellas Letras de Mahon (Mahon, 17-01-1837).

Una tercera carpeta identificada con la letra $C$ incluía certificados expedidos por influyentes personajes catalanes: Juan Francisco Bahi, catedrático de agricultura teórica y práctica y botánica en la Junta Nacional de Comercio de Barcelona ${ }^{95}$; Juan Agell, catedrático de mecánica de la Academia de Barcelona y secretario de la Sociedad Económica Barcelonesa de Amigos del País ${ }^{96}$; Carlos de Martí y Beseguin ${ }^{97}$ y José Aran y Barba, individuos de la referida

creación de la Sociedad Entomológica de Francia. Gouillard, J. (2004), Histoire des entomologistes français 1750-1950, París, Boubée, pp. 56-57.

92 Leon Dufour (1780-1865) entomólogo francés. Conoció a La Gasca en 1808, durante la visita que realizó a España acompañado por Etienne Geoffroy Saint-Hilaire, integrantes ambos del ejército de ocupación francés. Fue fundador de la Sociedad Entomológica de Francia. En la carta que firma recomendando a Graells se presenta como autor de la liquenografía de la Flore francaise publicada por Lamarck y de Candolle en 1805, así como de varios artículos sobre la anatomía de los insectos publicados en los Annales des Sciences Naturelles de Paris. Ibidem, pp. 26-28.

93 Alexandre Lefebvre (1798-1867) fue fundador de la sociedad entomológica francesa. Ejerció como secretario de la corporación entre 1831 y 1836 . Ibidem, pp. 56-57.

94 Pedro Giralt (1802-1879) era un educacionista y sacerdote catalán, residente en Uruguay, profesor de filosofía, geografía y latín que participó en la fundación del Ateneo de Montevideo. Aficionado a la zoología, remitía producciones de la naturaleza desde América.

95 Juan Francisco Bahi y Fonseca (1775-1841) enseñó agricultura en el jardín botánico de la Real Junta de Comercio y participó en la edición, en 1815, de Memorias de agricultura y artes, publicación en la que insertó numerosas colaboraciones. Se especializó en las enfermedades del olivo y en la creación de prados artificiales. En 1816 fue nombrado primer médico del hospital de Barcelona. EliAs DE Molins (1972), vol. I, pp. 188-193.

96 Juan Agell y Torrens (1809-1868) además de ser nombrado catedrático de mecánica en la Academia, en 1835, lo fue de física experimental en el Instituto Barcelonés (1841) y de química en la facultad de filosofía de Barcelona (1845). Se dedicó al estudio de la electricidad y de la telegrafía. Ibidem, pp. 1-4. La Gasca hace referencia expresa a él en una de sus cartas: «Basta que tu me recomiendes al Dr. Agell para que yo me interese por él, a lo que se añade ser catalán y discípulo de las escuelas de ese gran Consulado». Carta de La Gasca a Mariano de la Paz Graells. Madrid, 03-01-1836.

97 En abril de 1837 Carlos Martí leyó en la Academia una memoria acerca de las bases que pudieran adoptarse en el establecimiento de un sistema para regular los pesos y medidas en España. Ibidem, vol. II, pp. 97-98. 
Academia; José Boura, catedrático de química aplicada a las artes en Barcelona; Antonio Monmany, secretario de la Academia Nacional de Ciencias Naturales y Artes de Barcelona; Félix Janer, catedrático de clínica médica del Colegio de Medicina y Cirugía de Barcelona ${ }^{98}$; Juan Bautista Foix, catedrático de materia médica y química del referido Colegio ${ }^{99}$; Agustín Yáñez ${ }^{100}$, catedrático de historia natural en el Colegio Nacional de Farmacia de Barcelona y Ramón Taponera, notario y escribano de la Academia Territorial de Cataluña. Finalmente, la carpeta D contenía la relación de los méritos literarios contraídos por Graells en las cátedras de Gramática Castellana y Latina, Humanidades, Filosofía y Ciencias Médicas.

Ramón Ferrer y Garcés ${ }^{101}$, catedrático y secretario del Real Colegio de Medicina y Cirugía de Barcelona, es el encargado de pormenorizar los logros académicos del aspirante. Graells estudió tres años de gramática castellana y latina y dos de retórica en las Escuelas Pías de Barcelona, dos de filosofía escolástica en el Colegio Tridentino y uno de física experimental en la Casa Lonja con Pedro Vieta ${ }^{102}$. En 1827 recibió el grado de Bachiller en Artes en el Colegio de Medicina y Cirugía de Barcelona y se matriculó en los estudios de Medicina y Cirugía (siete años) que realizó de forma satisfactoria. Destacó por sus disecciones en las clases de anatomía y vendajes. Fue ayudante de

98 Félix Janer y Bertran (1771-1865) inició su carrera como catedrático de materia médica, terapeútica e higiene en la universidad de Cervera, en 1807. Posteriormente se encargó de las de medicina interna y su clínica en Barcelona y Madrid. Ibidem, vol. II, pp. 22-24.

99 Juan Bautista Foix y Gual (1780-1865), se dedicó fundamentalmente al estudio de la farmacología. Ibidem, vol. I, pp. 606-607.

100 Agustín Yañez y Girona (1789-1861) estudió humanidades y filosofía en el Seminario Conciliar, química, matemáticas y botánica en la Junta de Comercio y farmacia en el colegio de San Victoriano. Fue catedrático de farmacia, mineralogía y botánica y zoología médicas. Es autor del elogio histórico a La Gasca leído en la Academia de Barcelona en 1842. En 1820 publicó Lecciones de Historia Natural, primera obra didáctica española sobre el tema, que fue reeditada en 1845. En 1842 apareció su diccionario de historia natural y agricultura titulado Dios y sus obras, un texto redactado a partir de las obras de Buffon, Cuvier, Lacépède, Guérin y otros autores franceses. En él hizo un estudio de la lengua castellana y anotó sus deficiencias e inexactitudes en la materia abordada. Ibidem, vol. II, pp. 775-781.

${ }^{101}$ Ramón Ferrer y Garcés (1803-1872) era médico y enseñó medicina legal, toxicología e higiene pública en la universidad de Barcelona. Ibidem, vol I, pp. 594-595. La Gasca habla de él en su correspondencia: «El doctor Ferrer se ha portado perfectamente y parece está dispuesto a seguir el asunto (se refiere al de los baños de aguas termales) pero necesitan datos, datos y datos». Carta de La Gasca a Mariano de la Paz Graells. Madrid, 12-01-1837.

102 Pedro Vieta y Gibert (?-1856) fue vice-rector, decano y catedrático de filosofía en la universidad literaria de Barcelona y vice-presidente de la Academia de Medicina y Cirugía. Ibidem, vol. II, pp. 753-754. 
química médica, de terapéutica y materia médica (fue el depositario de las drogas y sustancias medicinales), de operaciones quirúrgicas y de obstetricia. En el cuarto año redactó una memoria sobre una rara enfermedad de las mujeres (no se dice cual). En quinto y sexto fue ayudante de bibliotecario. El doctor Ignacio Ameller ${ }^{103}$, director del centro, le encargó la redacción del diario clínico meteorológico, en el que anotaba diariamente las alteraciones atmosféricas y la influencia que éstas ejercían en los enfermos de las salas. El último año de su formación ayudó a Félix Janer en sus visitas clínicas.

\section{5. ¿UN CANDIDATO ADECUADO?}

Tras la reconstrucción de los acontecimientos que llevaron a Graells hasta su puesto de catedrático de zoología en la capital parece pertinente plantearse dos preguntas: ¿era el joven naturalista la apuesta ideal para cubrir el puesto vacante? ¿Hasta qué punto influyó su designación en el devenir de la disciplina en España?

Para responder a la primera de ellas hay que comenzar diciendo que, en zoología y en la España del XIX, el número de posibles candidatos era muy reducido. En el momento de someterse a evaluación, el bagaje científico de Graells distaba de ser escaso. Al margen de su formación académica e intelectual, el riojano ya desempeñaba entonces labores de catedrático de zoología en la academia barcelonesa, era responsable del incipiente museo de historia natural de la institución y autor de un calendario de flora, de varias memorias sobre insectos plaga y de dos artículos publicados en Francia. Frente a eso, Anastasio Chinchilla, el segundo aspirante, no poseía ningún trabajo sobre tema zoológico en el momento de aspirar a la vacante ${ }^{104}$. Su obra escrita se componía básicamente de memorias sanitarias inéditas y de algún artículo impreso con los primeros apuntes sobre la historia de la medicina en el paí $^{105}$. Su producción indirectamente relacionada con la historia natural se limi-

103 Ignacio Ameller y Ros (1769-1843) antes de ser nombrado catedrático en Barcelona, lo fue de materia médica en el Real Colegio de Cirugía de San Carlos de Madrid (1798), de química en la facultad de Salamanca (1799) y en el Colegio de Medicina de Burgos (1804). Ibidem, vol. I, pp. 65-67.

104 Más tarde, en 1844, Chinchilla publicaría una Memoria sobre los insectos perjudiciales a la agricultura y medios de destruirlos, trabajo premiado por la Sociedad Económica Valenciana. SALCEDO Y GINESTAL (1904), p. 155.

105 Las memorias médicas presentadas por Chinchilla antes de 1837 fueron: Baños y aguas minero-medicinales de Villavieja (Castellón de la Plana) (1829); De la topografía físico- 
taba a De la topografía fisico-médica de España en general, memoria que el autor estructuró en seis apartados dedicados respectivamente a la posición de España en el globo y su relación con la esfera celeste, las aguas comunes y las fuentes minerales, las piedras, tierras y minerales, las plantas, los animales y el hombre, secciones que en lo tocante a la botánica y la zoología no dejaban de ser meros listados de especies comunes ${ }^{106}$.

Esa escasez de candidatos hay que achacársela a la ausencia de una institucionalización de la zoología en España, materia que era cultivada de forma privada por personajes ilustrados, generalmente médicos, atraídos por la estimulante diversidad de los seres vivos. Desde el momento de la creación del Gabinete de Historia Natural en Madrid, en 1771, ya se había evocado la necesidad de enseñar la disciplina y para ello, Eugenio Izquierdo (1745-1813), vicedirector en tiempos de Pedro Franco Dávila (1713-1785) y director del centro tras su fallecimiento, se había formado en París. Sus prolongadas estancias en la capital francesa le impidieron impartir lecciones en el Museo. Sin embargo, poco a poco, las diferentes disciplinas iban encontrando acomodo en la institución y Clavijo y Fajardo (1730-1806), vicedirector del Gabinete, eligió la Histoire Naturelle de Buffon como libro guía para ilustrar la materia, obra que tradujo al castellano ${ }^{107}$. Con todo, durante el periodo Izquierdo-Clavijo la zoología no contó entre las enseñanzas impartidas en el Gabinete.

En 1815 Fernando VII ordenó la elaboración de un plan para la docencia de las ciencias naturales en el recién creado Real Museo de Ciencias Naturales, organismo que reunía al Gabinete de Historia Natural, el Jardín Botánico, el Laboratorio de Química y la Escuela de Mineralogía. El reglamento estableció cinco cátedras de las cuales dos estaban íntegramente dedicadas a la zoología, un generoso reparto poco acorde con la realidad científica del momento. De hecho, los dos puestos creados tardarían largo tiempo en cubrirse por falta

química de España en general (1829); Disertación histórico-fisico-médica de la villa de Cebreros (1830); Investigaciones físico-médicas de los meses de mayo, junio, julio y agosto de 1830 (Villa de Cebreros) (1830); Disertatio chimico-medica de lacte; de ejusdem speciebus, pro alimento et medicamento sumptis; et de illius praestantia, sive praejuditiis in medicina (1830). Sus artículos publicados en el Boletín de Medicina, Cirugía y Farmacia se titulaban: «El garrotillo» (1836); «Arnaldo de Vilanova» (1837); «Apuntes para servir de introducción a la Historia de la Medicina Española» (1837) y «Las suturas en cirugía» (1837). Ibidem, p. 75-122.

106 Ibidem, p. 81.

107 Clavijo y Fajardo, J. (2001), Prólogo a la traducción de la Historia Natural del conde de Buffon (Estudio preliminar de José Luis Prieto), La Orotava, Fundación Canaria Orotava de Historia de la Ciencia, 92 páginas. 
de candidatos apropiados ${ }^{108}$. El primero en ocuparse de la disciplina fue Tomás Villanova, médico de Valencia, que desde 1794 mantenía relaciones con el Gabinete como recolector de ejemplares. Su perseverancia ${ }^{109}$, su experiencia en otros museos, como el de Londres o el del príncipe de Parma ${ }^{110}$, y la finalización de su obra Ornitología ${ }^{111}$ le despejaron el camino hacia el ansiado puesto de profesor de zoología que pasó a ocupar en $1818^{112}$. Es muy poca la información de la que disponemos acerca de las clases impartidas por Villanova. Su primera lección de anatomía comparada tuvo lugar un 27 de noviembre de 1819, sesión en la que, siguiendo el método de Cuvier, fue oído por 51 alum$\operatorname{nos}^{113}$. En 1828 el número de estudiantes fue de $85^{114}$, cifra que se redujo a 31 en $1833^{115}$. Villanova era pues el primer catedrático de zoología en España, una disciplina surgida y asentada en el entorno museístico, que no realizaría su salto a la universidad hasta años más tarde, en pleno periodo Graells. Tras la muerte del valenciano resulta prematuro hablar de una primera generación de zoólogos españoles formada por él aunque, como ya ha sido dicho, uno de sus alumnos, Anastasio Chinchilla, se presentara como su legítimo sucesor a la cátedra.

La formación de Graells responde al esquema clásico del médico naturalista. Titulado en la única ciencia biológica que por aquel entonces podía asegurar un futuro profesional digno, el cultivo de las ciencias naturales fue en su caso consecuencia grata de una clara afición y del decido apoyo otorgado por su padre y por «su segundo padre». La influencia paterna en la obra de Mariano de la Paz es un asunto pendiente de resolver, así como la verdadera dimensión de la obra científica de Ignacio. Gran parte de su producción escrita permaneció inédita y tan solo un reducido número de trabajos llegó a la imprenta ${ }^{116}$. Valga como ejemplo la ingente cantidad de material recopilado sobre la distribución y clasificación de los insectos coleópteros, escritos que sin duda ayudaron a Ma-

108 AMNCN. Documentos del Real Gabinete de Historia Natural (1787-1813). Expediente 569 (1815).

109 Villanova, entre otras acciones encaminadas a obtener el puesto al que aspiraba, llegó a ofrecer un halcón naturalizado en prueba de su habilidad como taxidermista. AMNCN Documentos del Real Museo de Ciencias Naturales (1815-1845). Expediente 30 (13-02-1816).

110 BARREIRO (1992), p. 107.

111 Ibidem, p. 128.

112 AMNCN, Documentos del Real Museo de Ciencias Naturales (1815-1845). Expediente 73 (10-06-1818).

113 BARREIRO (1992), p. 132.

114 Ibidem, p. 158.

115 Ibidem, p. 169.

116 Elias de MOLInS (1972), p. 669. 
riano de la Paz en su carrera profesional. Los valiosos documentos, aparentemente recopilados en $1816^{117}$, siguen la clasificación propuesta por Latreille (1762-1833), sistema basado en el número de artejos presentes en el tarso de los insectos, cifra que puede oscilar entre uno (monómeros) y cinco (pentámeros) o variar entre los distintos pares de patas (heterómeros).

Lo que queda fuera de toda duda es la decidida voluntad de La Gasca a la hora de apadrinar a Mariano de la Paz y de guiarle hasta el epicentro de la historia natural hispana. En su calidad de mentor La Gasca no descuidó ninguna de sus obligaciones, ni la formación científica ni la promoción social. Tal tipo de relación genera una dependencia del pupilo respecto a su tutor, vínculo que a menudo va más allá del simple débito de favores y engendra una filiación intelectual. Es revelador que la cita a Linneo que La Gasca incluye en una de sus cartas, fechada en $1834^{118}$, aparezca mucho tiempo después en una de las mayores obras de Mariano de la Paz, su Fauna Mastodológica Ibérica ${ }^{119}$, publicada en 1897. Similar convergencia se encuentra en el interés que ambos tuvieron por la dimensión aplicada de la ciencia, utilitarismo que La Gasca considera propio de la tradición científica catalana:

« (...) catalán significa laborioso y aplicado, y la otra circunstancia dice ser la escuela mejor de conocimientos útiles que hay en la Nación. Además eso de catalán tiene en mi un (ilegible) de provincialismo, porque como mi razón se desplegó en Tarragona creo que soy tarragoní (sic). Sábelo para siempre, aunque no lo digas a nadie» ${ }^{120}$.

Graells fue quien trasvasó esa visión aplicada de la ciencia hasta Madrid, ciudad que había acumulado un notable retraso en el proceso de industrialización. El enfoque práctico en sus planteamientos primará a lo largo de su larga vida profe-

117 AMNCN, signatura ACN0332/004. Ignacio Graells. Apuntes científicos sobre diferentes géneros de Coleópteros.

118 «Sin embargo esto no es bastante, y así no satisface enteramente el espíritu, porque en los libros se presentan los seres en una serie lineal, que ciertamente no es el orden que sigue la naturaleza, sino en forma como de mares tanquam territorium in mappa geographica, según dijo sabiamente Linneo». Carta de La Gasca a Mariano de la Paz Graells. Saint-Heliers (Jersey), 18-04-1834.

119 «Sicut de plantis Linnaeus dixit. Animalia omnia utrinque, in Methodo affinitatem monstrant uti territorium in Mapa geographica». GRAELLS, M. P. (1897), Fauna Mastodológica Ibérica, Madrid, imprenta de Luis Aguado. El texto forma parte de la leyenda que acompaña la lámina que sirve para ilustrar la diversidad animal mediante la representación de islas y penínsulas en un símil geográfico.

120 Carta de La Gasca a Mariano de la Paz Graells. Madrid, 03-01-1836. 
sional, ya tenga entre manos problemas relacionados con la aclimatación de fauna $^{121}$, con la piscicultura ${ }^{122}$ o con el control de plagas de insectos ${ }^{123}$.

Por eso, y respondiendo a la segunda pregunta que planteábamos al inicio de esta discusión, podemos decir que, efectivamente, el padrinazgo de La Gasca influyó en la manera de hacer ciencia de Graells y en el devenir de la zoología en España, disciplina que por aquel entonces aún balbuceaba. La filiación entre ambos naturalistas parece trascender los contenidos para incidir igualmente en las prácticas, en una dinámica continuista que se relaciona con la noción de habitus propuesta por Bordieu ${ }^{124}$. Personas de un entorno social homogéneo poseen esquemas de obrar y pensar parecidos, lo que se traduce en que tienden a compartir estilos de vida similares.

Graells recogió el testigo del nepotismo directamente de la mano de La Gasca, y con él integró una forma de hacer ciencia en la que las relaciones personales tuvieron un especial peso en las decisiones académicas. A lo largo de su carrera Graells obró una excesiva personalización de sus proyectos, como ya ha sido demostrado con el de la aclimatación ${ }^{125}$, actitud que lleva asociados no pocos problemas, pues un contratiempo, un simple desánimo o la desaparición del interesado pueden acabar con todo. Según Ringer ${ }^{126}$, ese acatamiento voluntario de una tradición pasada responde a que las doctrinas se aceptan por el hecho de ser heredadas de precursores intelectuales, con los que además se comparte una orientación psicológica y una posición social y económica, en sintonía con el concepto de habitus previamente evocado.

Tradicionalmente, los historiadores de la ciencia consideran a Mariano de la Paz Graells como el último científico cortesano español. Durante los años centrales del periodo isabelino su figura se hizo omnipresente en los foros científicos de la capital y reprodujo la imagen del científico ilustrado próximo al poder, personalidad que Javier Puerto ha magistralmente desgranado en el botánico Casimiro Gómez Ortega (1741-1818). Disperso entre multitud de instituciones y tareas, su acción, básicamente diplomática y gestora, resulta

121 ARAGÓN (2005), 235 páginas.

122 GRAELLS, M. P. (1864), Manual de piscicultura o prontuario para servir de guía a los piscicultores de España y a los empleados de la administración pública de nuestras aguas dulces y saladas, Madrid, Aguado, XXIII + 264 páginas.

123 BuJ, A. (1996), El Estado y el control de plagas agrícolas, Madrid, Ministerio de Agricultura, Pesca y Alimentación, pp. 48-51.

124 Bordieu, P. (1988), La distinción, Madrid, Taurus, pp. 170-171.

125 ARAGÓN (2005), p. 200.

126 Ringer, F. (1995), El ocaso de los mandarines alemanes, Barcelona, Ediciones Pomares-Corredor, p. 21. 
poco efectiva en detrimento de la tarea científica profunda ${ }^{127}$. Curiosamente, tal actitud no está reñida con un reconocido prestigio, logrado a través de lo que el autor denomina «un complicado juego de espejos» ${ }^{128}$. Graells asume a la perfección ese papel de mandarín y basa su reconocimiento social en su nivel educativo y su estatus profesional ${ }^{129}$. Sus vínculos con el Estado y la Corona hicieron de él un personaje influyente e integró una élite con capacidad de decisión.

¿Por qué «el último»? Posiblemente porque las sociedades cambian más rápido que las personas y Graells tuvo que seguir la marcha de su tiempo. Desde su llegada al Museo Nacional de Ciencias Naturales, y a partir de 1845 en la universidad, Graells se convirtió en el principal profesor de zoología en España y por tanto en el instructor de la primera generación de zoólogos titulados. Tras su defenestración, en 1867, sus responsabilidades fueron repartidas entre varios naturalistas, lo que añade personajes a la escena: Miguel Colmeiro (1816-1901) asumió la dirección del Botánico, Lucas de Tornos (1803-1882) ${ }^{130}$ la de las colecciones de historia natural y Laureano Pérez Arcas (1824-1894) pasó a ocuparse del zoológico de aclimatación ${ }^{131}$. Algunos de sus discípulos iniciaron una diáspora que permitió extender la formación y la investigación en zoología a través del país, caso del cántabro Augusto González Linares (1845-1905), promotor de la Estación de Biología Marina de Santander ${ }^{132}$. Fuera o no consciente de ello, formara o no parte de sus planes, el mayor mérito de Graells fue el de lograr la institucionalización de la zoología en España y conseguir así esa masa crítica necesaria para que las reglas del juego cambiasen.

Fecha de recepción: 31 de mayo de 2006

Fecha de aceptación: 18 de julio de 2006

127 Puerto Sarmiento, F. J. (1992), Ciencia de cámara. Casimiro Gómez Ortega (17411818) el científico cortesano, Madrid, CSIC, pp. 97-98.

128 Ibidem, p. 101.

129 Ringer (1995), p. 23.

130 Lucas de Tornos también había sido discípulo y protegido de La Gasca, como relata Concepción Arenal en la semblanza que hizo del personaje tras su muerte. El joven Tornos, fiel asistente a los cursos de La Gasca, había llegado hasta la ciudad de Cádiz junto con la familia del botánico en 1823, tras el exilio de éste [ARENAL, C. (1883), «Biografía del Ilmo. Sr. D. Lucas de Tornos, Catedrático de número de la Universidad Central, fallecido en Madrid el 4 septiembre de 1882», Anales de la Sociedad Española de Historia Natural, XII, 41-52], La relación que mantuvieron posteriormente a lo largo de sus trayectorias vitales resta por precisar.

131 BARREIRO (1992), p. 262.

132 Ibidem, pp. 288-289. 
